\title{
SECURITY PROCEDURES FOR CLASSIFICATION MINING ALGORITHMS
}

\author{
Tom Johnsten \\ Computer Science Department \\ Western Illinois University \\ T-Johnsten@wiu.edu
}

\author{
Vijay V. Raghavan \\ Center for Advanced Computer Studies \\ University of Louisiana Lafayette \\ raghavan@louisiana.edu
}

\begin{abstract}
Classification mining algorithms can be used to discover protected values from non-protected data that are voluntarily released for mining purposes. We reported earlier on the development of an assessment algorithm, along with a set of security policies, for use in analyzing a protected data element's risk of disclosure with respect to decision-region based algorithms. This paper presents an extension of the previous work. Specifically, two algorithms, one for assessing a protected data element's risk of disclosure with respect to decision-region based algorithms and the other with respect to extended decision-region based algorithms, are developed. The former risk assessment algorithm has a relatively low execution time, but may not achieve an exact assessment of a protected data element's risk of disclosure. However, data collected from initial experiments indicate that the application of this particular assessment algorithm can lead to an exact assessment, under certain security policies.
\end{abstract}

Keywords: data mining, security, risk assessment, classification, database security.

\section{Introduction}

Nowadays companies and organizations frequently use data mining technology to analyze their stored data to discover valuable patterns or rules that can help them maintain their competitive edge. Recently, researchers within the information security community have begun to examine the impact of this technology on database security $[1,2,3,4,5]$.

The original version of this chapter was revised: The copyright line was incorrect. This has been corrected. The Erratum to this chapter is available at DOI: 10.1007/978-0-387-35587-0_24 
Our previous work focused on the disclosure of a protected data element through classification mining [5]. As part of that work, we identified a general category of classification mining algorithms, referred to as decision-region based, whose members possess a common set of properties related to the security risk assessment of a protected data element. Based on these properties, we developed a security risk assessment algorithm, along with a set of security policies, for use in analyzing decision-region based algorithms. We developed the risk assessment algorithm in the context of the relational data model. In the context of that model, we defined the terms protected attribute, non-protected attribute, and protected tuple. A protected attribute is the one that includes the protected data element (or attribute value) in its domain, a non-protected attribute contains no protected data elements in its domain, and a protected tuple contains a protected data element and at least one non-protected data element.

This paper introduces a broader category of classification mining algorithms, which we refer to as extended decision-region based, whose members possess a common set of properties that can be exploited during the assessment of the security risk of a protected data element. These algorithms, unlike those for decision-region based, are characterized as allowing for descriptions that are not entirely defined in terms of properties of the object. As a result, new assessment procedures must be developed with respect to this new category of classification algorithms. In this paper, we present an assessment algorithm, EXACT_OB2, designed specifically for use with extended decision-region based algorithms. We also present a new assessment algorithm, APPROX_EVAL, for use with decision-region based algorithms. The APPROX_EVAL algorithm is characterized has having a relatively low execution time, but may not achieve an exact assessment. However, data collected from initial experiments indicate that, in general, the application of the latter algorithm can lead to an exact assessment of the security risk associated with a protected data element, under certain security policies.

The rest of this paper is organized as follows. Section 1 presents an overview of our previous work, which we extend in this paper. Section 2 characterizes the extended decision-region based algorithms and presents an outline of the EXACT_OB2 algorithm. Section 3 presents an outline of the APPROX_EVAL algorithm; and, Section 4 describes the results of experiments that were conducted to assess the APPROX_EVAL's effectiveness. Finally, Section 5 presents the conclusions and discusses some future research projects.

\section{OVERVIEW OF PREVIOUS WORK}

We developed an algorithm, referred to as Orthogonal-Boundary (OB), that provides an exact assessment of the security risk of protected data elements with respect to decision-region based algorithms [5]. We define an exact assessment 
as one that guarantees the implementation of a security policy at the description space level. The notion of a description space level policy follows directly from the definition of a decision-region based classification algorithm.

A classification algorithm, $\mathrm{A}$, is a decision-region based algorithm if and only if the following two conditions are satisfied:

- Condition-1: It is possible to identify a priori a finite set of descriptions, $\mathrm{D}$, in terms of the attribute values present in an object $\mathrm{O}$ such that the particular description $d$ used by $A$ to classify $O$ is an element of $D$.

- Condition-2: The "predicted accuracy" of assigning an object satisfying a description $d \in D$ to a class $c$ is dependent on the distribution of class label $c$ relative to all other class labels among the objects that satisfy $d$ in the training set.

These conditions lead to the property that the effective assessment of the security threat from decision-region based algorithms requires the construction of a class-accuracy set for each description $\mathrm{d} \in \mathrm{D}$. We define a class-accuracy set as a collection of ordered pairs $\left(c_{i}, a_{i}\right)$, where $c_{i}$ is the $i^{\text {th }}$ attribute value (class label) in the domain of the protected attribute and $a_{i}$ is the predicted accuracy, according to the classification algorithm, of assigning to the protected tuple the class label $c_{i}$. Inference-based security policies may be applied at two levels. If it is known a priori that a particular description $d \in D$ will be selected relative to the protected tuple, then we can apply a policy just to that description. We refer to this type of policy as being defined at the description level. A more desirable approach is to ensure that no matter which $d \in D$ is chosen, the stated security policy is satisfied. We refer to this type of policy as being defined at the description space level. Given the above definitions and Condition-1, we concluded that inference-based security policies should be specified at the description space level, since description level policies require prior knowledge of which description will be chosen, which makes the results specific to a particular classification mining algorithm.

The OB algorithm has been designed for use with decision-region based algorithms that produce a specific type of description. In particular, those descriptions that are expressed as a logical conjunction of (attribute name, value) pairs and constructed from non-protected attribute values present in the protected tuple. The OB algorithm is, therefore, limited to decision-region based algorithms that divide the search space into hyper-rectangles. Those decisionregion based algorithms that divide the search space into "tilted" regions, such as decision-tree algorithms that use an attribute selection criterion based on a weighted sum of attribute values, and k-nearest neighbor classifiers which are defined in terms of a similarity measure, are outside the scope of OB.

The hyper-rectangular regions associated with a protected tuple are the logical conjunctions formed from one or more non-protected (attribute name, value) 
pairs that appear within the tuple. We refer to this set of descriptions as the description space, $D^{*}$, of the protected tuple. It follows from Condition- 1 that the assignment of a class label to a protected tuple is a label associated with a description $\mathrm{d} \in D^{*}$. There is no way to identify a priori the description $\mathrm{d} \in D^{*}$ that will be chosen by a classifier without making explicit assumptions about its operation. Thus, in general, it is necessary to inspect all descriptions that belong to a tuple's description space.

The risk assessment algorithm implements the description level protected rank security policy $[1,1]$. Given a class-accuracy set, a protected rank policy is satisfied if the ranked position of the protected data element is not within the non-secure range $[\mathrm{L}, \mathrm{U}]$, where $\mathrm{L}$ and $\mathrm{U}$ are positive integers such that $1 \leq \mathrm{L}$ $\leq \mathrm{U}$ and $\mathrm{L} \leq \mathrm{U} \leq\left|\left\{a_{1}, a_{2}, \ldots, a_{n}\right\}\right|$. To implement a protected rank policy it is necessary to compute a class-accuracy set for each individual description, $d$ $\in D^{*}$. The class-accuracy sets are computed based on Condition-2 as follows. Let $\mathrm{c}$ be a class label in the domain of a protected attribute. Given a description $\mathrm{d} \in D^{*}$, the predicted accuracy, $a$, of assigning the protected tuple $\mathrm{T}$ the label $c$ is the ratio of the number of tuples that are assigned label $c$ and satisfy $d$ to the number of tuples that satisfy $d$. This measure is equal to the classification accuracy measure defined in [6]. There are classification algorithms that satisfy Condition-1, but violate Condition-2. For instance, the CART decision-tree algorithm does not assume that the class labels are uniformly distributed, but instead computes predicted accuracy values using a weighted ratio based on prior probabilities [7].

A high-level outline of the OB algorithm, EXACT_OB1, is shown in Figure1. The result of executing this algorithm is the implicit or explicit inspection of each description belonging to the protected tuple's description space, $D^{*}$. The inspection process ensures that all descriptions, $\mathrm{d} \in D^{*}$, of a tuple's description space satisfy the stated description level security policy.

Unfortunately, the number of descriptions that belong to a protected tuple's description space is exponential in terms of the number of non-protected attributes. However, the recognition of a special type of description that we refer to as a zero description can significantly reduce the required number of inspections. The tuples that satisfy a zero description are characterized as having a class label that does not equal the protected data element. The occurrence of a zero description implies that there is no need to inspect any description that is a specialization since it will also be a zero description.

As shown in Figure-1, the EXACT_OB1 algorithm determines if a description, $\mathrm{d} \in D^{*}$, is a zero description. If $\mathrm{d}$ is a zero description it is placed on a list so that it is not directly examined by the algorithm, otherwise the algorithm determines if the description is non-secure. A non-secure description, $d$, is a description that violates the stated description level security policy. Currently, a description, $d$, is considered non-secure if it violates the protected rank policy 


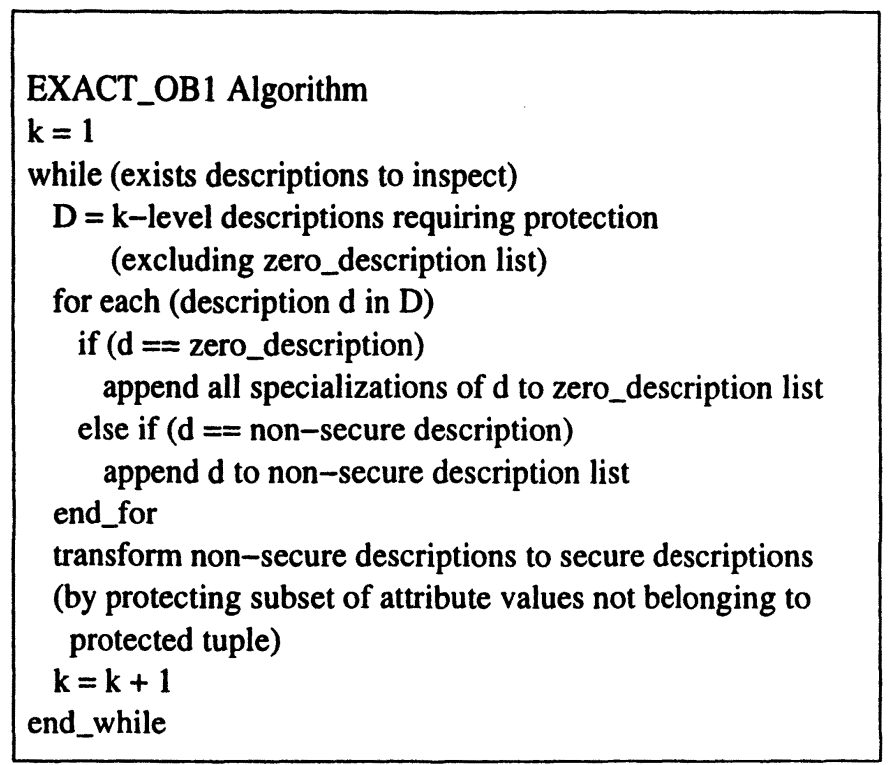

Figure 1. Orthogonal-Boundary Algorithm: EXACT_OB1.

$[1,1]$. The transformation of a non-secure description into a secure description is performed by concealing a subset of attribute values from the domain of nonprotected attributes that appear in the training set, but not in the protected tuple. The concealment of such values must decrease the number of tuples that satisfy the non-secure description with a class label equal to that of the protected data element, which can be repeated until there is no longer a violation of policy. This particular transformation has the following important property: the implementation of a policy, $P$, at the description level implies the implementation of $P$ at the description space level. In the context of EXACT_OB1, this property ensures the implementation of the protected rank policy $[1,1]$ at the description space level. It is important to note that EXACT_OB1 is only valid in the context of decision-region based algorithms. In the following section we present an algorithm that is designed for use with another category of classification algorithms, referred to as extended decision-region based.

\section{EXTENDED DECISION-REGION BASED ALGORITHMS}

We have generalized the EXACT_OB1 algorithm to provide an exact assessment of protected data elements with respect to extended decision-region based algorithms. A classification algorithm, A, is an extended decision-region based algorithm if and only if the following two conditions are satisfied: 
- Condition-1*: It is possible to identify a priori a finite set of descriptions, $D$, in terms of the attribute values present in an object $O$, and the attribute values present in a finite set of objects $O^{\prime}$ derived from $\mathrm{O}$, such that the particular description $\mathrm{d}$ used by $\mathrm{A}$ to classify $\mathrm{O}$ is an element of $\mathrm{D}$.

- Condition-2*: The predicted accuracy of assigning an object satisfying a description $d$ to a class $c$ is dependent on the distribution of class label $c$ relative to all other class labels among the objects that satisfy $d$ in the training set.

In Condition-1*, an object $O^{\prime}$ is derived from another object $O$ if it has at least one property in common with $\mathrm{O}$. The difference between Condition-1 and Condition $-1^{*}$ is that the latter condition allows for descriptions that are not entirely defined in terms of the properties of the target object. The second condition, Condition-2* , is identical to Condition-2 defined for decision-region based algorithms. Thus, a decision-region based algorithm is also an extended decision-region based algorithm.

An example of an extended decision-region based algorithm is C4.5's consult interpreter when applied to protected tuples with missing non-protected attribute values [8]. To illustrate, consider the protected tuple, (Cyl=4; Fuel=null; Tran=manu; Power=med; Mileage=null) where Mileage is the protected attribute, and the following class-accuracy values, 0 (low), .62 (med) and .38 (high) produced by C4.5. These confidence values were obtained through the assessment of the descriptions shown in Figure-2. It follows from the descriptions that there are two relevant protected tuples, $(C y l=4 ;$ Fuel=efi; Tran=manu; Power =med; Mileage $=$ null $)$ and $(C y l=4 ; F u e l=2-b b l ;$ Tran $=$ manu; Power $=$ med; Mileage $=n u l l)$. In general, there exists a relevant, or derived, protected tuple for each distinct value in the domain of a missing nonprotected attribute. The total number of relevant tuples is equal to the product of the domain sizes of the various non-protected attributes to which the missing attributes values belong. For instance, in the above example there are two relevant protected tuples since there is one missing non-protected attribute, Fuel, and its domain size is two, $\{2-b b l, e f i\}$. Similarly, if the protected tuple contains two missing non-protected attributes, Fuel and Power, the total number of relevant protected tuples is six since the domain size of Fuel is two and the domain size of Power is three, $\{l o w$, med, high $\}$. The description space of each relevant protected tuple must be inspected to determine if each description, $d$, satisfies the stated description level policy.

A high-level description of the EXACT_OB2 algorithm is shown in Figure-3. This algorithm is an extension of the EXACT_OB1 algorithm, and is designed for use with extended decision-region based algorithms. The EXACT_OB2 algorithm, like EXACT_OB1, has been developed in the context of descriptions that are represented by a logical conjunction of (attribute name, value) pairs. In 


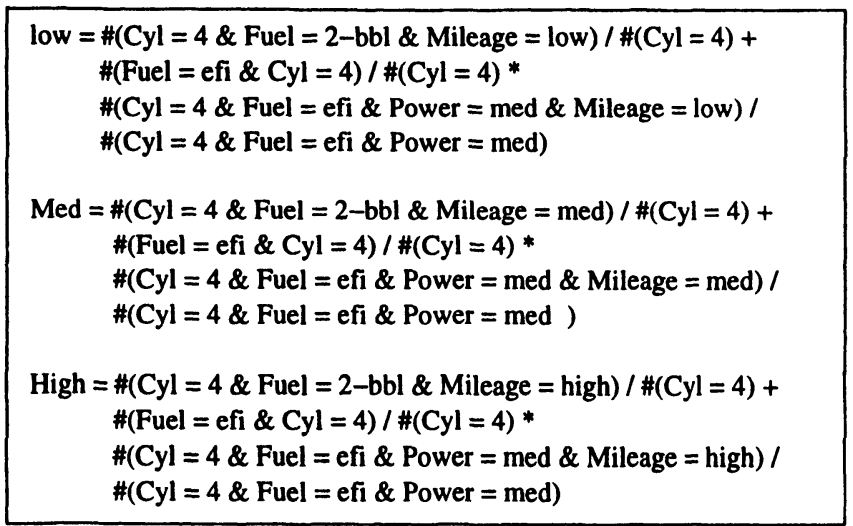

Figure 2. Example Descriptions.

\section{EXACT_OB2 Algorithm \\ PT = generate all relevant protected tuples \\ for each (protected tuple in PT) \\ apply EXACT_OB1 \\ end_for}

Figure 3. Orthogonal-Boundary Algorithm: Exact_OB2.

addition, we assume that the set of descriptions $\mathrm{D}$ is limited to those descriptions generated from the attribute values of the protected tuple as well as from values in the domain of the missing non-protected attributes of the protected tuple. Based on these restrictions, the result of executing the EXACT_OB2 algorithm is the inspection of the descriptions belonging to the description space of each relevant protected tuple. The structure of EXACT_OB2 is almost identical to the structure of EXACT_OB1. The only difference is that EXACT_OB2 inspects the description space of multiple protected tuples as opposed to a single protected tuple. The biggest limitation of EXACT_OB2, as well as EXACT_OB1, is the potentially high execution time. Specifically, the execution time of both algorithms is dependent upon the number of non-protected attributes and the occurrence of zero descriptions. As a result, we have developed an alternative assessment algorithm to approximate the exact assessment performed by EXACT_OB1. 


\section{APPROXIMATE ASSESSMENT ALGORITHM}

In this section, we present an assessment algorithm, which we will refer to as APPROX_EVAL. It approximates the exact assessment performed by EXACT_OB1. The idea behind APPROX_EVAL is to decrease the required execution time by reducing the size of the description space. One way to reduce the size of a description space is to transform a non-secure description into a secure description by concealing one or more non-protected attribute values. For example, if $(C y l=4)$ is a non-secure description with respect to the protected tuple, (Fuel=efi; $C y l=4$; Tran=manu; Mileage $=$ null), then the concealment of the non-protected attribute value, $C y l=4$, removes the description $C y l=4$ from $D^{*}$. In other words, the description cannot be used by a decision-region based algorithm to assign a value to the protected attribute.

The concealed value also removes from $D^{*}$ from the following descriptions, $($ Fuel=efi $\wedge C y l=4),(C y l=4 \wedge$ Tran=manu), and $(F u e l=e f i \wedge C y l=4 \wedge$ Tran=manu). As a result, there is no need to explicitly inspect any of these descriptions. However, as illustrated in the previous section, the concealment of non-protected attribute values may cause a classification algorithm, that normally adheres to the conditions of a decision-region based algorithm, to violate Condition-1 [5]. The APPROX_EVAL algorithm must, therefore, determine if a concealed non-protected attribute value is likely to result in such a violation. A high-level outline of APPROX.EVAL is shown in Figure-4. If it is determined that a violation is unlikely then the protected tuple is evaluated using the APPROX_OB algorithm; otherwise, it is evaluated using the EXACT_OB1 algorithm described in the previous section. The difference between the two algorithms is that APPROX_OB, unlike EXACT_OB1, transforms a non-secure description into a secure description by concealing one or more attribute values from the domain of non-protected attributes that appear in the protected tuple. In general, the execution time of APPROX_OB will be less than that of EXACT_OB1 due to the concealment of non-protected attribute values, and will also maximize the amount of available data.

The APPROX_EVAL algorithm may not achieve an exact assessment since it must predict the impact of a concealed non-protected attribute value on a decision-region based algorithm. However, the results obtained from the experimental investigation suggest that the prediction is likely to be consistent with an exact assessment.

\subsection{Predicted Accuracy Measure}

We have developed a measure to compute a class-accuracy set to determine if a concealed non-protected attribute value is likely to result in a violation of Condition-1. In this context, the class-accuracy set is computed independently of any specific description belonging to the protected tuple's description 


\section{APPROX_EVAL Algorithm}

prediction $=$ predict impact of concealed non-protected attribute values if (prediction $==$ violation of Condition-1)

apply EXACT_OB1 algorithm

else

apply APPROX_OB algorithm

Figure 4. Approximate Evaluation Algorithm (High-Level).

space. Instead, the predicted accuracy value $a$, with respect to a class label c, is computed based upon the degree to which the protected tuple's non-protected attribute values predict the class $\mathrm{c}$. The degree to which a non-protected attribute value predicts a protected attribute class depends upon the attribute value's relationship to the tuples that belong to the class as well as to the tuples that belong to other classes.

These relationships form the basis of our measure. Specifically, the proposed measure $M$, as shown in Figure-5, assigns a numerical value to a non-protected attribute value, $a$, that reflects the degree to which, $a$, predicts the protected attribute class $b$. The value $\mathrm{N}$ is equal to the number of tuples in the relation instance. The range of $M$ is the interval $[-1,1]$, where a value of positive (negative) one implies that the non-protected attribute value provides complete support (non-support) for the protected attribute class. The measure is comprised of two functions, class $(a, b)$ and nonclass $(a, b)$. The function, class $(a, b)$, captures the degree to which an implication of the form $\left(b \rightarrow a^{\prime}\right)$ weakens an implication of the form $(a \rightarrow b)$. The quantity \#(a,b) is the number of tuples in the relation instance with (attribute name, value) pairs A: $a$ and $\mathrm{B}: b$; and, the quantity $\#\left(a^{\prime}, b\right)$ is the number of tuples in the relation with (attribute name, value) pairs A: $a^{\prime}$ and $\mathrm{B}: b$. The symbol $a^{\prime}$ represents an element belonging to the set $\operatorname{Dom}(A)-a$. The other function, nonclass $(a, b)$, captures the degree to which an implication of the form $\left(b^{\prime} \rightarrow a^{\prime}\right)$ weakens an implication of the form $\left(a \rightarrow b^{\prime}\right)$. The symbol $b^{\prime}$ represents an element belonging to the set $\operatorname{Dom}(B)-b$. The quantity \#(a,bi $\left.b_{i}\right)$ represents the number of tuples in the relation instance with (attribute name, value) pairs A: $a$ and $\mathrm{B}: b_{i}$, where $b_{i}$ is an instance of B such that $b_{i} \neq b$; and, \# $\left(a^{\prime}, b_{i}\right)$ represents the number of tuples in the relation instance with (attribute name, value) pairs $\mathrm{A}: a^{\prime}$ and $\mathrm{B}: b_{i}$. Finally, the condition expressed as cond -1 is satisfied if $\#\left(a, b_{i}\right) / \#\left(a^{\prime}, b_{i}\right)=1$ and $\#\left(a, b_{j}\right)$ $/ \#\left(a^{\prime}, b_{j}\right)<1$ for all $\mathrm{j} \neq \mathrm{i}$ where $b_{i}, b_{j} \in \operatorname{Dom}(B)-b$. Intuitively, the function, nonclass $(a, b)$, computes the degree of predictability associated with the class, $b^{\prime}$, that the attribute value $a$ predicts to the greatest extent. 


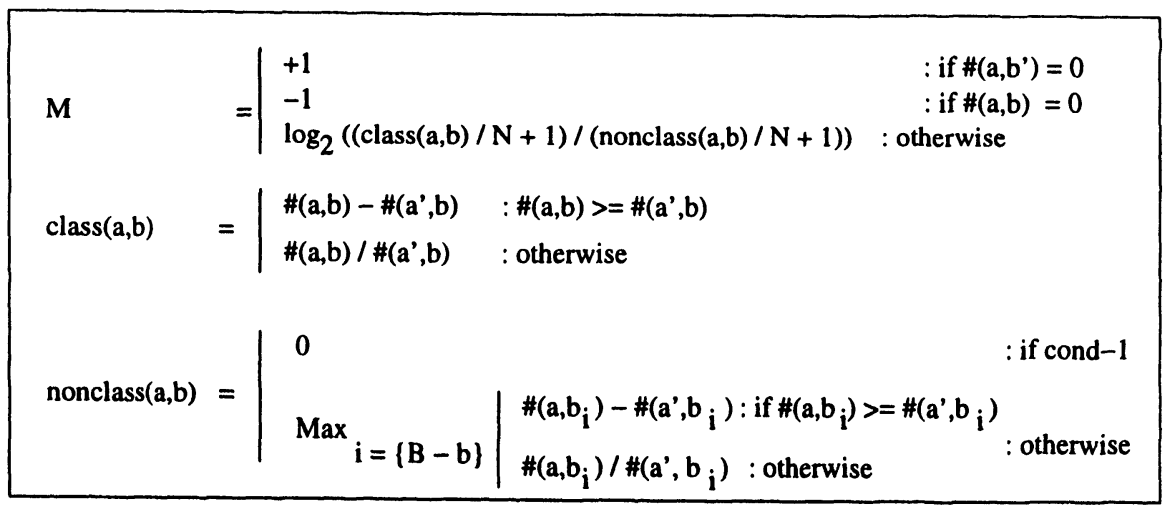

Figure 5. Predicted Accuracy Measure $M$.

The definitions of $\operatorname{class}(a, b)$ and nonclass $(a, b)$ represent the concept of a non-protected attribute's relevance and non-relevance odds, respectively. This type of relevance relationship has been used previously in information retrieval systems to determine a document term's degree of importance in distinguishing relative and non-relative documents [9].

\subsection{APPROX EVAL Algorithm}

The measure $M$ is used to compute the class-accuracy set of a protected tuple. Specifically, the assigned weights that correspond to a class, $c$, are summed together to produce the corresponding predicted accuracy value. For example, suppose the application of $M$ with respect to the protected tuple, ( $C y l=6 ;$ Fuel=2-bbl; Tran=manu; Power=low; Mileage=null), produces the results shown in Figure- 6 . In this instance, the predicted class-accuracy set is \{(low, -1.09), (med, -.255), (high, -.946)\}. In general, a computed predicted accuracy value represents the degree to which the protected tuple predicts the corresponding protected data element.

The class-accuracy set is used in conjunction with the Protected Minimum Range (PMR) security policy to determine if there is a violation of Condition1. The application of a PMR policy is dependent upon the rank position of the predicted accuracy value, $a_{i}$, of the protected data element relative to the other predicted accuracy values. If $a_{i}$ is the maximum predicted accuracy value, then the $p m r$ value is the difference between $a_{i}$ and the next largest predicted accuracy value $a_{k}$; otherwise, it is the difference between $a_{i}$ and and the maximum predicted accuracy value.

As shown in Figure-7, if $p m r$ is greater than some established threshold, then the APPROXEVAL algorithm evaluates the protected data element using 
Low: $\{$ Cyl=6: .1699; Fuel=2-bbl: -.0458 ; Tran=manu: -.2157 ; Power=low: -1$\}$

Med: $\{$ Cyl=6: -.1699 ; Fuel=2-bbl: -.0771 , Tran=manu: 0; Power=low: -.0079$\}$

High: $\{$ Cyl=6: -1 ; Fuel=2-bbl: .0458; Tran = manu: 0; Power=low: .0079 $\}$

Figure 6. Application of Measure M.

APPROX_EVAL Algorithm

compute measure $M$ with respect to protected tuple

compute corresponding class-accuracy set

pmr $=$ apply Protected Minimum Range policy to class-accuracy set

if (pmr >= threshold-value)

apply EXACT_OB1 algorithm

else

apply APPROX_OB algorithm

Figure 7. Approximate Evaluation Algorithm (Detail-Level).

the EXACT_OB1 algorithm; otherwise, the protected data element is evaluated using the APPROX_OB algorithm.

\section{EXPERIMENTAL INVESTIGATION}

Experiments were conducted to validate the use of the APPROX EVAL algorithm in implementing the description space level protected rank policy $[1,1]$. In conducting our initial experiments, we restricted the evaluation to C4.5's decision-tree classifier and interpreter [8]. The experiments utilized ten distinct data sets, each of which contained at least ten non-protected attribute values and a single protected attribute with a domain size of five. All of the relations were constructed through the application of the Synthetic Classification Data Set (SCDS) program [10]. A total of fifty-one protected tuples were used in the investigation. Twenty-one of the protected tuples violated the description space level policy $[1,1]$.

Figure-8 shows the $p m r$ value computed for each of the fifty-one protected tuples. The APPROX_OB algorithm was applied to each protected tuple. The application of APPROX_OB resulted in the transformation of a protected tuple $T$ into a protected tuple $T^{\prime}$ that, in general, contained one or more concealed non-protected attribute values. The transformed protected tuples, $T^{\prime}$, were 


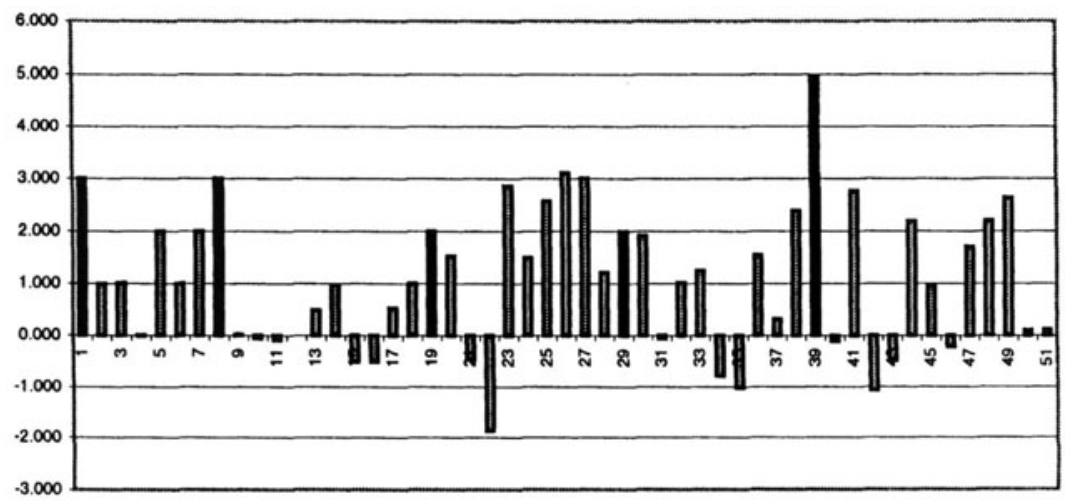

Figure 8. Computed pmr Values for Protected Tuples.

classified using C4.5's consult interpreter. In order to assess the generality of the APPROXEVAL algorithm two distinct decision-tree models where generated for each relation instance. One model was constructed using the gain attribute selection criterion, while the other model was constructed using the gain ratio attribute selection criterion. We identified those protected tuples that violated the description space level protected rank policy $[1,1]$. There were a total of five such tuples, five with respect to the gain ratio criterion and one with respect to the gain criterion. These five tuples are shown as solid-filled columns in Figure-8.

The experimental results suggest that a $p m r$ value greater than or equal to two identifies a potential violation of Condition-1. Unfortunately, such a $p m r$ value may produce false positives. It appears that the occurrence of false positives may be related to the percentage of non-relevant attributes contained within a relation.

\section{CONCLUSION AND FUTURE WORK}

In this paper we identified a new category of classification mining algorithms, referred to as extended decision-region based, and presented a security risk assessment algorithm, EXACT_OB2, designed specifically for use with this category of algorithms. In addition, we proposed an alternative assessment algorithm, APPROX EVAL, designed for use with decision-region based algorithms. This particular assessment algorithm is characterized as having a relatively low execution time, but may not achieve an exact assessment.

We have several additional research projects planned with respect to this challenging research area. Our immediate plans include further verification of experimental results using additional extended decision-region based algorithms and data sets, extending the security assessments to include continuously 
valued attributes, and the development of additional security assessments for other groups of mining algorithms.

\section{References}

[1] Chang, L. and Moskowitz, I. (1998). Parsimonious Downgrading and Decision Trees Applied to the Inference Problem. Proceedings of New Security Paradigms, pp. 82-89.

[2] Chang, L. and Moskowitz, I. (2000). An Integrated Framework for Database Privacy Protection. Proceedings of the fourteenth Annual IFIP WG 11.3 Working Conference on Database Security.

[3] Clifton, C. and Marks, D. (1996). Security and Privacy Implications of Data Mining. 1996 SIGMOD Workshop on Research Issues on Data Mining and Knowledge Discovery, pp. 15-19.

[4] Clifton, C. (1999). Protecting Against Data Mining Through Samples. Proceedings of the Thirteenth Annual IFIP WG 11.3 Working Conference on Database Security, pp. 193-207.

[5] Johnsten, T. and Raghavan, V. (1999). Impact of Decision-Region Based Classification Mining Algorithms on Database Security. Proceedings of the Thirteenth Annual IFIP WG 11.3 Working Conference on Database Security, pp. 177-191.

[6] Holsheimer, M. and Siebes, A. (1994). Data Mining: The Search for Knowledge in Databases. Report CS-R9406. CWI. Amsterdam, The Netherlands.

[7] Steinberg, D. and Colla, P. (1997). CART User Manual. Salford Systems, San Diego, CA.

[8] Quinlan, J. (1993). C4.5: Programs For Machine Learning. Morgan Kaufmann, San Mateo, CA.

[9] Robertson, S. and Jones, K. (1976). Relevance Weighting for Search Terms. Journal of the American Society for Information Science, May-June, pp. 129-145.

[10] Synthetic Classification Data Sets, http:/lfas.sfu.ca/cs/people/Grad Students/melli/SCDS/intro.html. 